
COMMUNICATIONS

ISSN 2056-9890

Received 23 December 2017

Accepted 5 January 2018

Edited by P. Dastidar, Indian Association for the Cultivation of Science, India

Keywords: crystal structure; palladium-catalysed reactions; Buchwald-Hartwig amination; pyrazine; morpholine.

CCDC reference: 1585321

Supporting information: this article has supporting information at journals.iucr.org/e

\section{Crystal structure of 4-(pyrazin-2-yl)morpholine}

\author{
Siva Sankar Murthy Bandaru, ${ }^{\mathrm{a}}$ Anant Ramakant Kapdi ${ }^{\mathrm{b}}$ and Carola Schulzke ${ }^{\mathrm{a} *}$
}

'Institut für Biochemie, Ernst-Moritz-Arndt Universität Greifswald, Felix-Hausdorff-Strasse 4, D-17487 Greifswald, Germany, and ${ }^{\mathbf{b}}$ Department of Chemistry, Institute of Chemical Technology, Nathalal Parekh Road, Matunga, Mumbai 400 019, India. *Correspondence e-mail: carola.schulzke@uni-greifswald.de

The molecular structure of the title compound, $\mathrm{C}_{8} \mathrm{H}_{11} \mathrm{~N}_{3} \mathrm{O}$, is nearly planar despite the chair conformation of the morpholine moiety. In the crystal, the molecules form sheets parallel to the $b$ axis, which are supported by non-classical hydrogen-bonding interactions between $\mathrm{C}-\mathrm{H}$ functionalities and the $\mathrm{O}$ atom of morpholine and the 4-N atom of pyrazine, respectively. The title compound crystallizes in the monoclinic space group $P 2_{1} / c$ with four molecules in the unit cell.

\section{Chemical context}

The potential applications of aryl and heteroaryl amines in chemistry, materials science and pharmaceutical industries encourages research into the formation of $\mathrm{C}-\mathrm{N}$ bonds (Rappoport, 2007; Lawrence, 2004, Weissermel \& Arpe 1997). $\mathrm{N}$-Heteroarylmorpholine moieties are prevalent in biologically active molecules such as medicines for the treatment of schizophrenia or type-2 diabetes mellitus (BartoloméNebreda et al., 2014). In this context we are engaged in the synthesis of a library of heterocyclic amine derivatives. In course of these investigations, pure crystalline 4-(pyrazin-2yl)morpholine was isolated with the crystals being obtained upon purification by column chromatography.

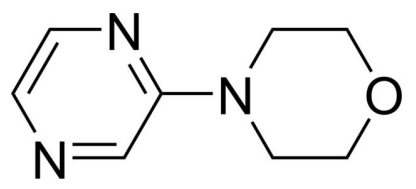

\section{Structural commentary}

4-(Pyrazin-2-yl)morpholine (Fig. 1) crystallizes in the monoclinic space group $P 2_{1} / c$ with four molecules in the unit cell. There are reports in the literature of the molecular structures of compounds in which the morpholine nitrogen atom is coupled to the carbon atom of a non-annelated $N$-heterocyclic

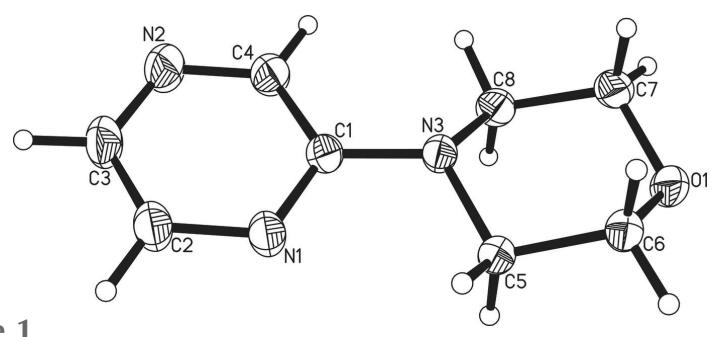

Figure 1

The molecular structure of the title compound, showing the atom labelling and $50 \%$ probability displacement ellipsoids. 
pyridine (Dahlgren et al., 2012; Horton et al., 2012; Huth et al., 2007; Klauschenz et al., 1994; Li et al., 2014, Reck et al., 1992) or pyrimidine (Cheprakova et al., 2014; García et al., 2009; Gorbunov et al., 2013; Hansen \& Geffken, 2012; Vinogradova et al., 2016). For pyrazine as the heterocycle, however, (to the best of our knowledge and after conducting a database search, see $\$ 4$ ) the present work constitutes the first structural report even though the title compound itself has been known since 1969 (Abe et al., 1969).

The orientation of the morpholine ring, in its typical chair conformation, relative to the aromatic plane can be either more or less in plane (e.g. Vinogradova et al., 2016), tilted around the $\mathrm{N}-\mathrm{C}$ bond (e.g. Li et al., 2014), bent away from the aromatic plane (e.g. Hansen \& Geffken, 2012) or a combination of the latter two (e.g. Reck et al., 1992), depending on the other substituents on the heterocycle. In the present case, a morpholine ring is as much aligned with the N1/N2/C1-C4 plane as its conformation allows, with the carbon $\mathrm{C} 8$ showing the largest distance from the plane of 0.414 (1) $\AA$. This distance is shorter than for any of the pyridine or pyrimidine derivatives without morpholine disorder from the reports mentioned above. The largest deviation from the plane of the pyrizine atoms was found to be 0.013 (1) $\AA$ for C1 and C4.
The quality of the crystallographic data allowed the hydrogen atoms to be located and refined entirely freely without any constraints or restraints. The information content of the metrical parameters involving the hydrogen atoms, including non-classical hydrogen-bonding interactions, is therefore comparably high. The $\mathrm{C}-\mathrm{H}$ distances for the aromatic atoms are 0.999 (15) $\AA$ for C2, 0.976 (16) $\AA$ for C3 and 0.962 (16) $\AA$ for $\mathrm{C} 4$. The methylene protons are in a distance range from their parent carbon atoms of 0.978 (14) to 1.016 (14) $\AA$ with a tendency for the longer $\mathrm{C}-\mathrm{H}$ bond to be for the hydrogen atom in the axial position [only $\mathrm{C} 7$ is an exception with distances of 1.003 (14) $\AA$ for the axial and 1.005 (14) $\AA$ for the equatorial position]. All $\mathrm{C}-\mathrm{C}, \mathrm{C}-\mathrm{N}$ and $\mathrm{C}-\mathrm{O}$ bond lengths are within the commonly observed ranges.

\section{Supramolecular features}

In the crystal, the molecules form sheets parallel to the $b$ axis supported by non-classical hydrogen-bonding interactions (Fig. 2, Table 1). In each molecule, the pyrazine ring is tilted slightly out of the general orientation of the sheets and the direction of the rotation alternates between adjacent rows (protruding along the $b$ axis) as well as between adjacent
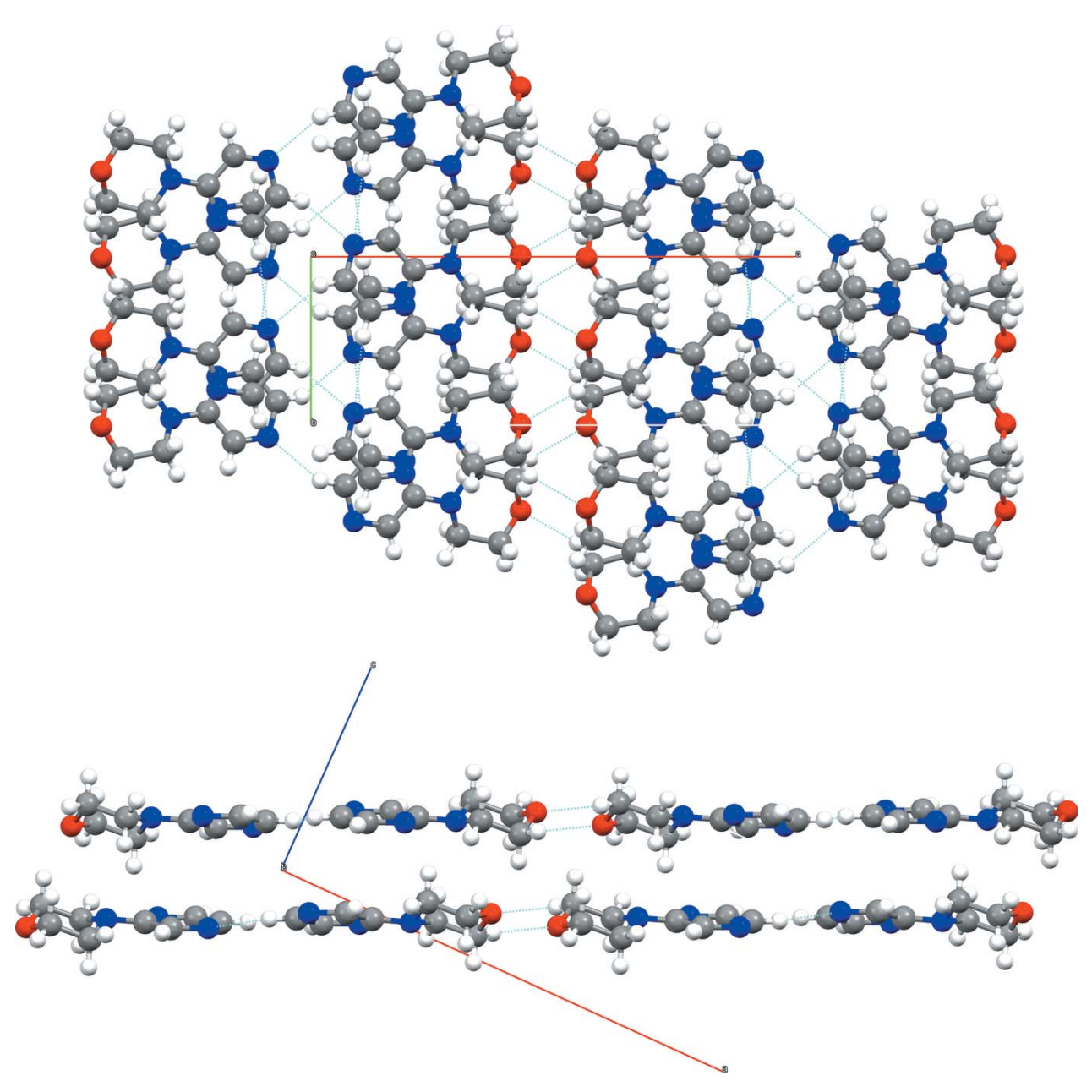

Figure 2

The crystal packing (Mercury; Macrae et al., 2006) viewed (top) along the $c$ axis and (bottom) along the $b$ axis showing the layered arrangement and the non-classical hydrogen-bonding interactions (Table 1) between the molecules of a sheet. 
Table 1

Hydrogen-bond geometry $\left(\AA,^{\circ}\right)$.

\begin{tabular}{lllll}
\hline$D-\mathrm{H} \cdots A$ & $D-\mathrm{H}$ & $\mathrm{H} \cdots A$ & $D \cdots A$ & $D-\mathrm{H} \cdots A$ \\
\hline $\mathrm{C} 6-\mathrm{H} 6 A \cdots \mathrm{O} 1^{\mathrm{i}}$ & $0.988(14)$ & $2.561(14)$ & $3.4841(16)$ & $155.5(10)$ \\
$\mathrm{C} 3-\mathrm{H} 3 \cdots \mathrm{N} 2^{\mathrm{ii}}$ & $0.976(16)$ & $2.670(16)$ & $3.5723(19)$ & $153.9(13)$ \\
$\mathrm{C} 2-\mathrm{H} 2 \cdots \mathrm{N} 2^{\mathrm{iii}}$ & $0.999(15)$ & $2.743(15)$ & $3.6840(19)$ & $157.2(11)$ \\
$\mathrm{C} 4-\mathrm{H} 4 \cdots \mathrm{N} 1^{\text {iv }}$ & $0.962(16)$ & $2.787(16)$ & $3.6775(18)$ & $154.3(11)$ \\
\hline
\end{tabular}

Symmetry codes: (i) $-x+1, y+\frac{1}{2},-z+\frac{1}{2}$; (ii) $-x+2, y+\frac{1}{2},-z+\frac{3}{2}$; (iii) $x, y+1, z$; (iv) $x, y-1, z$.

layers with an angle of $17.95^{\circ}$ between the two variants of torsion.

Within the sheets, each molecule forms hydrogen-bonding interactions to six surrounding molecules. These are donor interactions involving $\mathrm{C} 2[\mathrm{C} 2-\mathrm{H} 2 \cdots \mathrm{N} 2(x, y+1, z)], \mathrm{C} 3[\mathrm{C} 3-$ $\mathrm{H} 3 \cdots \mathrm{N} 2\left(-x+2, y+\frac{1}{2},-z+\frac{3}{2}\right], \mathrm{C} 4[\mathrm{C} 4-\mathrm{H} 4 \cdots \mathrm{N} 1(x, y-1, z)]$ and $\mathrm{C} 6\left[\mathrm{C} 6-\mathrm{H} 6 A \cdots \mathrm{O} 1\left(-x+1, y+\frac{1}{2},-z+\frac{1}{2}\right)\right]$ and acceptor interactions involving $\mathrm{N} 1[\mathrm{~N} 1 \cdots \mathrm{H} 4-\mathrm{C} 4(x, y+1, z)], \mathrm{N} 2$ $\left[\mathrm{N} 2 \cdots \mathrm{H} 2-\mathrm{C} 2(x, y-1, z), \mathrm{N} 2 \cdots \mathrm{H} 3-\mathrm{C} 3\left(-x+2, y+\frac{1}{2},-z+\frac{3}{2}\right]\right.$ and $\mathrm{O} 1\left[\mathrm{O} 1 \cdots \mathrm{H} 6 A-\mathrm{C} 6\left(1-x, \frac{1}{2}+y, \frac{1}{2}-z\right)\right]$.

No $\pi-\pi$ interactions are apparent between the sheets, with the closest distance between aromatic ring centroids being 4.2470 (11) Å while two sheets are $3.564 \AA$ apart.

\section{Synthesis and crystallization}

The synthesis was carried out under an inert gas atmosphere $\left(\mathrm{N}_{2}\right)$ applying the typical Schlenk line procedures. To an ovendried Schlenk tube $(25 \mathrm{~mL})$ were added $\mathrm{Pd}(\mathrm{OAc})_{2}(1 \mathrm{~mol} \%$, $0.0024 \mathrm{~g}$ ) and PTABS (phosphatriazene adamantyl butane

Table 2

Experimental details.

\begin{tabular}{|c|c|}
\hline \multicolumn{2}{|l|}{ Crystal data } \\
\hline Chemical formula & $\mathrm{C}_{8} \mathrm{H}_{11} \mathrm{~N}_{3} \mathrm{O}$ \\
\hline$M_{\mathrm{r}}$ & 165.20 \\
\hline Crystal system, space group & Monoclinic, $P 2_{1} / c$ \\
\hline Temperature $(\mathrm{K})$ & 170 \\
\hline$a, b, c(\AA)$ & $17.069(3), 5.9278(12), 7.8053$ (16) \\
\hline$\beta\left({ }^{\circ}\right)$ & $90.54(3)$ \\
\hline$V\left(\AA^{6}\right)$ & $789.7(3)$ \\
\hline$Z$ & 4 \\
\hline Radiation type & Mo $K \alpha$ \\
\hline$\mu\left(\mathrm{mm}^{-1}\right)$ & 0.10 \\
\hline Crystal size (mm) & $0.38 \times 0.31 \times 0.26$ \\
\hline \multicolumn{2}{|l|}{ Data collection } \\
\hline Diffractometer & Stoe IPDS2T \\
\hline $\begin{array}{l}\text { No. of measured, independent and } \\
\text { observed }[I>2 \sigma(I)] \text { reflections }\end{array}$ & $6583,1666,1285$ \\
\hline$R_{\text {int }}$ & 0.044 \\
\hline$(\sin \theta / \lambda)_{\max }\left(\AA^{-1}\right)$ & 0.634 \\
\hline \multicolumn{2}{|l|}{ Refinement } \\
\hline$R\left[F^{2}>2 \sigma\left(F^{2}\right)\right], w R\left(F^{2}\right), S$ & $0.032,0.081,1.01$ \\
\hline No. of reflections & 1666 \\
\hline No. of parameters & 153 \\
\hline H-atom treatment & All $\mathrm{H}$-atom parameters refined \\
\hline$\Delta \rho_{\max }, \Delta \rho_{\min }\left(\mathrm{e} \AA^{-3}\right)$ & $0.19,-0.18$ \\
\hline
\end{tabular}

Computer programs: $X$-AREA (Stoe \& Cie, 2010), SHELXT2016 (Sheldrick, 2015a), SHELXL2016 (Sheldrick, 2015b), XP in SHELXTL (Sheldrick, 2008), Mercury (Macrae et al., 2006) and CIFTAB (Sheldrick, 2008). saltone; $2 \mathrm{~mol} \%, 0.00586 \mathrm{~g}$ ) and a nitrogen atmosphere was generated. To this were added $3 \mathrm{~mL}$ of dry DMF followed by the addition of 2-chloropyrazine $(0.086 \mathrm{~mL}, 1 \mathrm{mmol}), 1.5$ equivalents of triethylamine $(0.3 \mathrm{~mL}, 1.5 \mathrm{mmol})$ and 1.1 equivalent of morpholine $(0.1 \mathrm{~mL}, 1.1 \mathrm{mmol})$. The suspension was stirred at room temperature for $4 \mathrm{~h}$ and progress of the reaction was monitored by TLC. After completion of the reaction, the crude product was purified and isolated by column chromatography in an EtOAc:hexane (1:3) solvent system. The final sharp colourless needles $(0.124 \mathrm{mg}$, $0.83 \mathrm{mmol}, 83 \%$ ) were obtained directly after the column purification step by crystallizing from the eluent. The mounted crystal was a block cut from a large needle. The compound has a low melting point of only $318 \mathrm{~K}$ and the crystals were stored in the fridge until they were measured.

${ }^{1} \mathrm{H}$ NMR (300 MHz, chloroform- $d$ ) $\delta$ ppm 3.51-3.63 ( $m$, $4 \mathrm{H}), 3.79-3.90(m, 4 \mathrm{H}), 7.90(d, J=2.64 \mathrm{~Hz}, 1 \mathrm{H}), 8.14(d, J=$ $7.6 \mathrm{~Hz}, 1 \mathrm{H}), 9.61(d, J=7.8 \mathrm{~Hz}, 1 \mathrm{H}) .{ }^{13} \mathrm{C} \mathrm{NMR}(75 \mathrm{MHz}$, chloroform- $d) \delta$ ppm $45.18(s, 1 \mathrm{C}) 66.93(s, 1 \mathrm{C}) 77.42(s, 1 \mathrm{C})$ $77.84(s, 1 \mathrm{C}) 131.33(s, 1 \mathrm{C}) 133.98(s, 1 \mathrm{C}) 142.16(s, 1 \mathrm{C}) 155.48$ $(s, 1 C)$. ESI-MS $(m / z)=166.17(M+\mathrm{H})^{+}, 167.22(M+2 \mathrm{H})^{2+}$ (cf. Graham et al., 2011).

\section{Refinement}

Crystal data, data collection and structure refinement details are summarized in Table 2. All hydrogen atoms were located and refined freely without any constraints or restraints.

\section{Funding information}

ARK and CS acknowledges 'The Alexander von Humboldt Foundation' for the research cooperation programme and the equipment grant to ARK. CS gratefully acknowledges funding from the ERC for the project MocoModels.

\section{References}

Abe, Y., Shigeta, Y., Uchimaru, F. \& Okada, S. (1969). Daiichi Seiyaku Co., Ltd. . JP44012739B.

Bartolomé-Nebreda, J. M., Delgado, F., Martín-Martín, M. L., Martínez-Viturro, C. M., Pastor, J., Tong, H. M., Iturrino, L., Macdonald, G. J., Sanderson, W., Megens, A., Langlois, X., Somers, M., Vanhoof, G. \& Conde-Ceide, S. (2014). J. Med. Chem. 57, 41964212.

Cheprakova, E. M., Verbitskiy, E. V., Ezhikova, M. A., Kodess, M. I., Pervova, M. G., Slepukhin, P. A., Toporova, M. S., Kravchenko, M. A., Medvinskiy, I. D., Rusinov, G. L. \& Charushin, V. N. (2014). Russ. Chem. Bull. 63, 1350-1358.

Dahlgren, M. K., Garcia, A. B., Hare, A. A., Tirado-Rives, J., Leng, L., Bucala, R. \& Jorgensen, W. L. (2012). J. Med. Chem. 55, 1014810159.

García, A., Insuasty, B., Cobo, J. \& Glidewell, C. (2009). Acta Cryst. C65, o598-0600.

Gorbunov, E. B., Novikova, R. K., Plekhanov, P. V., Slepukhin, P. A., Rusinov, G. L., Rusinov, V. L., Charushin, V. N. \& Chupakhin, O. N. (2013). Chem. Heterocycl. Compd, 49, 766-775.

Graham, T. H., Liu, W. \& Shen, D.-M. (2011). Org. Lett. 13, 62326235.

Hansen, F. K. \& Geffken, D. (2012). J. Heterocycl. Chem. 49, 321-328. 
Horton, P. N., Mohamed, S. K., Soliman, A. M., Abdel-Raheem, E. M. M. \& Akkurt, M. (2012). Acta Cryst. E68, o885-o886.

Huth, S. L., Hursthouse, M. B. \& Withnell, J. (2007). University of Southampton, Crystal Structure Report Archive, 355.

Klauschenz, E., Hagen, V., Wiesner, B., Hagen, A., Reck, G. \& Krause, E. G. (1994). Eur. J. Med. Chem. 29, 175-184.

Lawrence, S. A. (2004). Editor. Amines: Synthesis, Properties, Application. Cambridge University Press.

Li, Q., Zhang, S.-Y., He, G., Ai, Z., Nack, W. A. \& Chen, G. (2014). Org. Lett. 16, 1764-1767.

Macrae, C. F., Edgington, P. R., McCabe, P., Pidcock, E., Shields, G. P., Taylor, R., Towler, M. \& van de Streek, J. (2006). J. Appl. Cryst. 39, 453-457.
Rappoport, Z. (2007). Editor. The Chemistry of Anilines. Weinheim: Wiley-VCH.

Reck, G., Hagen, V. \& Bannier, G. (1992). Pharmazie, 47, 852856.

Sheldrick, G. M. (2008). Acta Cryst. A64, 112-122.

Sheldrick, G. M. (2015a). Acta Cryst. A71, 3-8.

Sheldrick, G. M. (2015b). Acta Cryst. C71, 3-8.

Vinogradova, K. A., Krivopalov, V. P., Nikolaenkova, E. B., Pervukhina, N. V., Naumov, D. Y., Boguslavsky, E. G. \& Bushuev, M. B. (2016). Dalton Trans. 45, 515-524.

Weissermel, K. \& Arpe, H. J. (1997). Editors. Industrial Organic Chemistry. Weinheim: Wiley-VCH.

Stoe \& Cie (2010). X-AREA. Stoe \& Cie, Darmstadt, Germany. 


\section{supporting information}

Acta Cryst. (2018). E74, 137-140］[https://doi.org/10.1107/S2056989018000312]

\section{Crystal structure of 4-(pyrazin-2-yl) morpholine}

\section{Siva Sankar Murthy Bandaru, Anant Ramakant Kapdi and Carola Schulzke}

Computing details

Data collection: $X$-AREA (Stoe \& Cie, 2010); cell refinement: $X$-AREA (Stoe \& Cie, 2010); data reduction: $X$-AREA (Stoe $\&$ Cie, 2010); program(s) used to solve structure: SHELXT2016 (Sheldrick, 2015a); program(s) used to refine structure: SHELXL2016 (Sheldrick, 2015b); molecular graphics: XP in SHELXTL (Sheldrick, 2008) and Mercury (Macrae et al., 2006); software used to prepare material for publication: CIFTAB (Sheldrick, 2008).

4-(Pyrazin-2-yl)morpholine

Crystal data

$\mathrm{C}_{8} \mathrm{H}_{11} \mathrm{~N}_{3} \mathrm{O}$

$M_{r}=165.20$

Monoclinic, $P 2_{1} / c$

$a=17.069(3) \AA$

$b=5.9278(12) \AA$

$c=7.8053(16) \AA$

$\beta=90.54(3)^{\circ}$

$V=789.7(3) \AA^{3}$

$Z=4$

Data collection

Stoe IPDS2T

diffractometer

Radiation source: fine-focus sealed tube

Detector resolution: 6.67 pixels $\mathrm{mm}^{-1}$

$\omega$ scans

6583 measured reflections

1666 independent reflections

\section{Refinement}

Refinement on $F^{2}$

Least-squares matrix: full

$R\left[F^{2}>2 \sigma\left(F^{2}\right)\right]=0.032$

$w R\left(F^{2}\right)=0.081$

$S=1.01$

1666 reflections

153 parameters

0 restraints
$F(000)=352$

$D_{\mathrm{x}}=1.389 \mathrm{Mg} \mathrm{m}^{-3}$

Mo $K \alpha$ radiation, $\lambda=0.71073 \AA$

Cell parameters from 7070 reflections

$\theta=5.2-53.6^{\circ}$

$\mu=0.10 \mathrm{~mm}^{-1}$

$T=170 \mathrm{~K}$

Block, colourless

$0.38 \times 0.31 \times 0.26 \mathrm{~mm}$

1285 reflections with $I>2 \sigma(I)$

$R_{\text {int }}=0.044$

$\theta_{\text {max }}=26.8^{\circ}, \theta_{\min }=3.6^{\circ}$

$h=-21 \rightarrow 21$

$k=-7 \rightarrow 7$

$l=-9 \rightarrow 9$

Hydrogen site location: difference Fourier map

All $\mathrm{H}$-atom parameters refined

$w=1 /\left[\sigma^{2}\left(F_{\mathrm{o}}^{2}\right)+(0.0391 P)^{2}+0.1242 P\right]$

where $P=\left(F_{\mathrm{o}}{ }^{2}+2 F_{\mathrm{c}}{ }^{2}\right) / 3$

$(\Delta / \sigma)_{\max }<0.001$

$\Delta \rho_{\max }=0.19 \mathrm{e} \AA^{-3}$

$\Delta \rho_{\min }=-0.18 \mathrm{e} \AA^{-3}$ 


\section{Special details}

Geometry. All esds (except the esd in the dihedral angle between two 1.s. planes) are estimated using the full covariance matrix. The cell esds are taken into account individually in the estimation of esds in distances, angles and torsion angles; correlations between esds in cell parameters are only used when they are defined by crystal symmetry. An approximate (isotropic) treatment of cell esds is used for estimating esds involving l.s. planes.

Fractional atomic coordinates and isotropic or equivalent isotropic displacement parameters $\left(\hat{A}^{2}\right)$

\begin{tabular}{lllll}
\hline & $x$ & $y$ & $z$ & $U_{\text {iso }} * / U_{\text {eq }}$ \\
\hline O1 & $0.56805(5)$ & $0.49320(14)$ & $0.28629(10)$ & $0.0288(2)$ \\
N1 & $0.80725(6)$ & $0.78798(17)$ & $0.57424(13)$ & $0.0273(2)$ \\
N2 & $0.91130(6)$ & $0.4299(2)$ & $0.62453(16)$ & $0.0389(3)$ \\
N3 & $0.71160(5)$ & $0.53996(16)$ & $0.47126(12)$ & $0.0225(2)$ \\
C1 & $0.78753(7)$ & $0.57747(19)$ & $0.53119(14)$ & $0.0227(3)$ \\
C2 & $0.87897(7)$ & $0.8160(2)$ & $0.64356(18)$ & $0.0327(3)$ \\
C3 & $0.93002(8)$ & $0.6409(2)$ & $0.66999(19)$ & $0.0370(3)$ \\
C4 & $0.84119(7)$ & $0.4000(2)$ & $0.55398(17)$ & $0.0318(3)$ \\
C5 & $0.66501(6)$ & $0.73760(19)$ & $0.42267(15)$ & $0.0226(3)$ \\
C6 & $0.57955(7)$ & $0.6729(2)$ & $0.40626(15)$ & $0.0255(3)$ \\
C7 & $0.61043(7)$ & $0.3001(2)$ & $0.34360(16)$ & $0.0271(3)$ \\
C8 & $0.69742(7)$ & $0.3467(2)$ & $0.35819(15)$ & $0.0258(3)$ \\
H6B & $0.5600(7)$ & $0.621(2)$ & $0.5203(17)$ & $0.028(3)^{*}$ \\
H5B & $0.6834(7)$ & $0.804(2)$ & $0.3125(16)$ & $0.023(3)^{*}$ \\
H5A & $0.6694(7)$ & $0.853(2)$ & $0.5118(17)$ & $0.027(3)^{*}$ \\
H8B & $0.7235(8)$ & $0.210(3)$ & $0.4052(17)$ & $0.031(4)^{*}$ \\
H7B & $0.5899(7)$ & $0.248(2)$ & $0.4570(17)$ & $0.029(3)^{*}$ \\
H6A & $0.5487(8)$ & $0.802(2)$ & $0.3624(16)$ & $0.028(3)^{*}$ \\
H4 & $0.8289(8)$ & $0.248(3)$ & $0.5193(18)$ & $0.038(4)^{*}$ \\
H7A & $0.6002(8)$ & $0.177(2)$ & $0.2579(17)$ & $0.028(3)^{*}$ \\
H8A & $0.7193(8)$ & $0.380(2)$ & $0.2402(17)$ & $0.034(4)^{*}$ \\
H2 & $0.8931(8)$ & $0.975(3)$ & $0.6735(17)$ & $0.035(4)^{*}$ \\
H3 & $0.9810(9)$ & $0.670(3)$ & $0.723(2)$ & $0.049(4)^{*}$ \\
& & & & \\
\hline
\end{tabular}

Atomic displacement parameters $\left(\AA^{2}\right)$

\begin{tabular}{lllllll}
\hline & $U^{11}$ & $U^{22}$ & $U^{33}$ & $U^{12}$ & $U^{13}$ & $U^{23}$ \\
\hline O1 & $0.0287(5)$ & $0.0263(5)$ & $0.0310(4)$ & $-0.0026(4)$ & $-0.0104(3)$ & $0.0010(3)$ \\
N1 & $0.0217(5)$ & $0.0252(5)$ & $0.0348(6)$ & $-0.0007(4)$ & $-0.0041(4)$ & $-0.0036(4)$ \\
N2 & $0.0255(6)$ & $0.0337(6)$ & $0.0571(7)$ & $0.0040(5)$ & $-0.0100(5)$ & $0.0021(5)$ \\
N3 & $0.0200(5)$ & $0.0191(5)$ & $0.0283(5)$ & $0.0005(4)$ & $-0.0037(4)$ & $-0.0012(4)$ \\
C1 & $0.0199(5)$ & $0.0240(6)$ & $0.0242(5)$ & $-0.0006(4)$ & $0.0009(4)$ & $0.0009(4)$ \\
C2 & $0.0251(6)$ & $0.0303(7)$ & $0.0427(7)$ & $-0.0023(5)$ & $-0.0065(5)$ & $-0.0042(6)$ \\
C3 & $0.0219(6)$ & $0.0382(8)$ & $0.0507(8)$ & $-0.0016(6)$ & $-0.0094(6)$ & $-0.0013(6)$ \\
C4 & $0.0259(6)$ & $0.0247(6)$ & $0.0448(7)$ & $0.0013(5)$ & $-0.0057(5)$ & $0.0011(5)$ \\
C5 & $0.0215(6)$ & $0.0212(5)$ & $0.0251(6)$ & $0.0003(5)$ & $-0.0023(4)$ & $0.0003(5)$ \\
C6 & $0.0222(6)$ & $0.0245(6)$ & $0.0297(6)$ & $-0.0005(5)$ & $-0.0039(5)$ & $0.0006(5)$ \\
C7 & $0.0300(6)$ & $0.0228(6)$ & $0.0282(6)$ & $-0.0044(5)$ & $-0.0054(5)$ & $0.0005(5)$ \\
C8 & $0.0286(6)$ & $0.0209(6)$ & $0.0279(6)$ & $0.0001(5)$ & $-0.0024(5)$ & $-0.0023(5)$ \\
& & & & & &
\end{tabular}


Geometric parameters $\left(\AA,{ }^{\circ}\right)$

\begin{tabular}{|c|c|c|c|}
\hline $\mathrm{O} 1-\mathrm{C} 7$ & $1.4238(15)$ & $\mathrm{C} 3-\mathrm{H} 3$ & $0.976(16)$ \\
\hline $\mathrm{O} 1-\mathrm{C} 6$ & $1.4307(14)$ & $\mathrm{C} 4-\mathrm{H} 4$ & $0.962(16)$ \\
\hline $\mathrm{N} 1-\mathrm{C} 1$ & $1.3345(15)$ & $\mathrm{C} 5-\mathrm{C} 6$ & $1.5126(16)$ \\
\hline $\mathrm{N} 1-\mathrm{C} 2$ & $1.3441(16)$ & $\mathrm{C} 5-\mathrm{H} 5 \mathrm{~B}$ & $0.999(13)$ \\
\hline $\mathrm{N} 2-\mathrm{C} 4$ & $1.3244(16)$ & $\mathrm{C} 5-\mathrm{H} 5 \mathrm{~A}$ & $0.978(14)$ \\
\hline $\mathrm{N} 2-\mathrm{C} 3$ & $1.3381(18)$ & C6-H6B & $1.002(13)$ \\
\hline $\mathrm{N} 3-\mathrm{C} 1$ & $1.3917(15)$ & C6-H6A & $0.988(14)$ \\
\hline $\mathrm{N} 3-\mathrm{C} 5$ & $1.4639(15)$ & $\mathrm{C} 7-\mathrm{C} 8$ & $1.5135(17)$ \\
\hline N3-C8 & $1.4648(14)$ & C7-H7B & $1.003(14)$ \\
\hline $\mathrm{C} 1-\mathrm{C} 4$ & $1.4051(17)$ & C7-H7A & $1.005(14)$ \\
\hline $\mathrm{C} 2-\mathrm{C} 3$ & $1.3693(19)$ & $\mathrm{C} 8-\mathrm{H} 8 \mathrm{~B}$ & $0.995(15)$ \\
\hline $\mathrm{C} 2-\mathrm{H} 2$ & $0.999(15)$ & $\mathrm{C} 8-\mathrm{H} 8 \mathrm{~A}$ & $1.016(14)$ \\
\hline $\mathrm{C} 7-\mathrm{O} 1-\mathrm{C} 6$ & $109.07(9)$ & $\mathrm{N} 3-\mathrm{C} 5-\mathrm{H} 5 \mathrm{~A}$ & $109.7(8)$ \\
\hline $\mathrm{C} 1-\mathrm{N} 1-\mathrm{C} 2$ & $116.31(10)$ & $\mathrm{C} 6-\mathrm{C} 5-\mathrm{H} 5 \mathrm{~A}$ & $107.7(8)$ \\
\hline $\mathrm{C} 4-\mathrm{N} 2-\mathrm{C} 3$ & $116.61(11)$ & $\mathrm{H} 5 \mathrm{~B}-\mathrm{C} 5-\mathrm{H} 5 \mathrm{~A}$ & $108.3(10)$ \\
\hline $\mathrm{C} 1-\mathrm{N} 3-\mathrm{C} 5$ & $117.46(9)$ & $\mathrm{O} 1-\mathrm{C} 6-\mathrm{C} 5$ & $111.74(10)$ \\
\hline $\mathrm{C} 1-\mathrm{N} 3-\mathrm{C} 8$ & $118.39(9)$ & $\mathrm{O} 1-\mathrm{C} 6-\mathrm{H} 6 \mathrm{~B}$ & $108.0(7)$ \\
\hline $\mathrm{C} 5-\mathrm{N} 3-\mathrm{C} 8$ & $112.55(9)$ & $\mathrm{C} 5-\mathrm{C} 6-\mathrm{H} 6 \mathrm{~B}$ & $109.3(7)$ \\
\hline $\mathrm{N} 1-\mathrm{C} 1-\mathrm{N} 3$ & $117.76(10)$ & $\mathrm{O} 1-\mathrm{C} 6-\mathrm{H} 6 \mathrm{~A}$ & $106.3(7)$ \\
\hline $\mathrm{N} 1-\mathrm{C} 1-\mathrm{C} 4$ & $120.38(11)$ & $\mathrm{C} 5-\mathrm{C} 6-\mathrm{H} 6 \mathrm{~A}$ & $110.1(8)$ \\
\hline $\mathrm{N} 3-\mathrm{C} 1-\mathrm{C} 4$ & $121.79(11)$ & $\mathrm{H} 6 \mathrm{~B}-\mathrm{C} 6-\mathrm{H} 6 \mathrm{~A}$ & $111.4(10)$ \\
\hline $\mathrm{N} 1-\mathrm{C} 2-\mathrm{C} 3$ & $122.88(12)$ & $\mathrm{O} 1-\mathrm{C} 7-\mathrm{C} 8$ & $111.85(10)$ \\
\hline $\mathrm{N} 1-\mathrm{C} 2-\mathrm{H} 2$ & $115.3(8)$ & $\mathrm{O} 1-\mathrm{C} 7-\mathrm{H} 7 \mathrm{~B}$ & $110.0(8)$ \\
\hline $\mathrm{C} 3-\mathrm{C} 2-\mathrm{H} 2$ & $121.9(8)$ & $\mathrm{C} 8-\mathrm{C} 7-\mathrm{H} 7 \mathrm{~B}$ & $109.9(7)$ \\
\hline $\mathrm{N} 2-\mathrm{C} 3-\mathrm{C} 2$ & $121.23(12)$ & $\mathrm{O} 1-\mathrm{C} 7-\mathrm{H} 7 \mathrm{~A}$ & $106.9(7)$ \\
\hline $\mathrm{N} 2-\mathrm{C} 3-\mathrm{H} 3$ & $119.1(10)$ & $\mathrm{C} 8-\mathrm{C} 7-\mathrm{H} 7 \mathrm{~A}$ & $110.3(8)$ \\
\hline $\mathrm{C} 2-\mathrm{C} 3-\mathrm{H} 3$ & $119.7(10)$ & $\mathrm{H} 7 \mathrm{~B}-\mathrm{C} 7-\mathrm{H} 7 \mathrm{~A}$ & $107.8(11)$ \\
\hline $\mathrm{N} 2-\mathrm{C} 4-\mathrm{C} 1$ & $122.53(12)$ & $\mathrm{N} 3-\mathrm{C} 8-\mathrm{C} 7$ & $110.14(10)$ \\
\hline $\mathrm{N} 2-\mathrm{C} 4-\mathrm{H} 4$ & $115.9(9)$ & $\mathrm{N} 3-\mathrm{C} 8-\mathrm{H} 8 \mathrm{~B}$ & $110.2(8)$ \\
\hline $\mathrm{C} 1-\mathrm{C} 4-\mathrm{H} 4$ & $121.6(9)$ & $\mathrm{C} 7-\mathrm{C} 8-\mathrm{H} 8 \mathrm{~B}$ & $108.3(8)$ \\
\hline $\mathrm{N} 3-\mathrm{C} 5-\mathrm{C} 6$ & $109.88(10)$ & $\mathrm{N} 3-\mathrm{C} 8-\mathrm{H} 8 \mathrm{~A}$ & $109.6(8)$ \\
\hline $\mathrm{N} 3-\mathrm{C} 5-\mathrm{H} 5 \mathrm{~B}$ & $111.4(7)$ & $\mathrm{C} 7-\mathrm{C} 8-\mathrm{H} 8 \mathrm{~A}$ & $109.6(8)$ \\
\hline $\mathrm{C} 6-\mathrm{C} 5-\mathrm{H} 5 \mathrm{~B}$ & 109.7 (7) & $\mathrm{H} 8 \mathrm{~B}-\mathrm{C} 8-\mathrm{H} 8 \mathrm{~A}$ & $108.9(11)$ \\
\hline
\end{tabular}

Hydrogen-bond geometry $\left(\AA,{ }^{\circ}\right)$

\begin{tabular}{lllll}
\hline$D-\mathrm{H} \cdots A$ & $D-\mathrm{H}$ & $\mathrm{H} \cdots A$ & $D \cdots A$ & $D-\mathrm{H} \cdots A$ \\
\hline $\mathrm{C} 6-\mathrm{H} 6 A \cdots \mathrm{O} 1^{\mathrm{i}}$ & $0.988(14)$ & $2.561(14)$ & $3.4841(16)$ & $155.5(10)$ \\
$\mathrm{C} 3-\mathrm{H} 3 \cdots \mathrm{N} 2^{\mathrm{ii}}$ & $0.976(16)$ & $2.670(16)$ & $3.5723(19)$ & $153.9(13)$ \\
$\mathrm{C} 2-\mathrm{H} 2 \cdots \mathrm{N} 2^{\mathrm{iii}}$ & $0.999(15)$ & $2.743(15)$ & $3.6840(19)$ & $157.2(11)$ \\
$\mathrm{C} 4-\mathrm{H} 4 \cdots \mathrm{N} 1^{\text {iv }}$ & $0.962(16)$ & $2.787(16)$ & $3.6775(18)$ & $154.3(11)$ \\
\hline
\end{tabular}

Symmetry codes: (i) $-x+1, y+1 / 2,-z+1 / 2$; (ii) $-x+2, y+1 / 2,-z+3 / 2$; (iii) $x, y+1, z$; (iv) $x, y-1, z$. 